\title{
Georgios N. Yannakakis and Julian Togelius: Artificial Intelligence and Games
}

\section{Springer, 2018, Print ISBN: 978-3-319-63518-7, Online ISBN: 978-3-319-63519-4, https://doi.org/10.1007/978-3-319-63519-4}

\section{Pablo García-Sánchez ${ }^{1}$}

Published online: 11 September 2018

(c) Springer Science+Business Media, LLC, part of Springer Nature 2018

Computer games have been tied to the research of Artificial Intelligence since its beginning. ${ }^{1}$ Research on AI has not only benefited from using classic games, such as chess or Go, as a basis for advancement, but recently modern videogames such as StarCraft have become de facto testbeds to test new techniques and methods [4]. In addition, the videogame sector has established itself as one of the great pillars of the current entertainment industry, competing in revenues with other markets, such as the film sector, and even allowing the professionalization of players.

Nowadays, many universities are offering students curricula related to the development of videogames, so there is a need for new teaching materials that cover the different branches of this discipline, such as Software Engineering, Testing, Quality Control, Artificial Intelligence and Player Modelling. Unfortunately, there is a lack of textbooks covering the last two branches. For this reason, Professors Georgios N. Yannakais (Director of the Institute of Digital Games at the University of Malta) and Julian Togelius (Co-director of the Game Innovation Lab of the New York University) have decided to write this book. Both are experts in the field of computational intelligence in games, with joint publications for more than 10 years. They have also organized conferences such as the IEEE CIG (now IEEE COG) ${ }^{2}$ or AIIDE, ${ }^{3}$ and have occupied the position of editors of the journal "IEEE Transactions on Computational Intelligence and AI in Games", among others.

There are currently other books that deal with AI in games, such as Millington and Funges's "Artificial Intelligence for Games" [3], or are exclusive to specific

\footnotetext{
1 This is the basis of the plot for the film "Wargames" (1983).

2 http://www.ieee-cig.org/.

3 https://www.aaai.org/Conferences/AIIDE/aiide.php.

Pablo García-Sánchez

pablo.garciasanchez@uca.es

1 Department of Computer Engineering, ESI, University of Cádiz, Avenida de la Universidad 10, Campus Universitario de Puerto Real, 11519 Cádiz, Spain
} 
development engines (such as Unity [1]). However, what distinguishes this book from the others is that it not only focuses on programming AIs for NPC (Non Playable Characters) in videogames, but also extends its use to other areas such as content generation (for example, terrain generation or interactive fiction) or human player modelling (such as sentiment analysis or playing behaviour).

The book is structured in three parts. The first one offers an overview of the current state of the art, and the different existing techniques that can be applied, such as Evolutionary Computation or Machine Learning, explaining the most important methods of each field and showing examples of use in real games.

The second part shows how to use AI in Games in three areas: game design, human player modelling and content generation, offering different classification taxonomies. Particularly interesting is the classification of games based on observability, stochasticity and granularity over time and which technique is most suited in each situation. As in the previous section, each topic is explained with a large number of examples, including games available on the market, and not just limited to NPC modelling. For example, it describes the use of Self-Organizing Maps (SOM) to model and classify the types of players who play the game Tomb Raider: Underworld [2].

The last section of the book discusses possible areas of exploration in the field of computational intelligence in games, and how the different areas and topics can interact, as well as the frontiers of the current research on game AI.

The book is easy to read and follow, as each part is well structured and offers summaries at the end of each chapter. It also includes more than 800 bibliographic references that will be useful to those interested in learning more about any of the topics described. A great choice is that the same videogame (Ms. Pacman) is used to explain each of the techniques described. In addition, it has a large number of colored figures that help to strengthen the knowledge, either by describing an outline or by showing the application of a technique in a specific game. Finally, the book has an associated website (http://gameaibook.org) that offers additional material, such as slides, references and software. At the end of each chapter a list of possible exercises to be developed by the reader is available, inviting to implement the different techniques explained. These exercises are not solved, instead they can be offered to students as assignments. The exercise materials are available on the book's website, along with links to frameworks and datasets that the reader can use and are updated often.

This book is mostly aimed at students and teachers of Artificial Intelligence and Games to be used as a textbook for subjects related to the topic, but also serves as a reference manual for researchers. Although readers of this journal will not encounter many examples of how to use GP directly, they may find the described techniques for evaluating agents or content generators relevant (for example, to create proper fitness functions). It is also important to note that this book is not only oriented to the academic world. Professional videogame developers can also take advantage of its content, due to its more introductory approach and its lack of scientific jargon, although a small base, such as an introductory AI course, would be recommended. 


\section{References}

1. R. Barrera, A.S. Kyaw, T.N. Swe, Unity 2017 AI Programming (Packt Publishing, Birmingham, 2018)

2. T. Mahlmann, A. Drachen, J. Togelius, A. Canossa, G.N. Yannakakis, Predicting player behavior in tomb raider: underworld, in Proceedings of the 2010 IEEE Conference on Computational Intelligence and Games, CIG 2010, Copenhagen, Denmark, 18-21 August, 2010, ed. by G.N. Yannakakis, J. Togelius (IEEE, 2010), pp. 178-185

3. I. Millington, J. Funge, Artificial Intelligence for Games (CRC Press, Boca Raton, 2009)

4. S. Ontañón, G. Synnaeve, A. Uriarte, F. Richoux, D. Churchill, M. Preuss, A survey of real-time strategy game AI research and competition in starcraft. IEEE Trans. Comput. Intell. AI Games 5(4), 293-311 (2013) 